\title{
CHARACTERIZATION OF SIGNAL CONDUCTION ALONG DEMYELINATED AXONS BY ACTION- POTENTIAL-ENCODED SECOND HARMONIC GENERATION
}

\author{
ZHI-HUI LUO, JIANG-XU CHEN, YI-MEI HUANG, HONG-QIN YANG*, \\ JU-QIANG LIN, HUI LI and SHU-SEN XIE \\ Institute of Laser and Optoelectronics Technology \\ Fujian Provincial Key Laboratory for Photonics Technology \\ Key Laboratory of Optoelectronic Science and Technology \\ for Medicine of Ministry of Education \\ Fujian Normal University, Fuzhou 35000\%, P. R. China \\ *hqyang@fjnu.edu.cn
}

Received 31 May 2013

Accepted 15 September 2013

Published 2 December 2013

\begin{abstract}
Action-potential-encoded optical second harmonic generation (SHG) has been recently proposed for use in detecting the axonal damage in patients with demyelinating diseases. In this study, the characterization of signal conduction along axons of two different levels of demyelination was studied via a modified Hodgkin-Huxley model, because some types of demyelinating disease, i.e., primary progressive and secondary progressive multiple sclerosis, are difficult to be distinguished by magnetic resonance imaging (MRI), we focused on the differences in signal conduction between two different demyelinated axons, such as the first-level demyelination and the secondlevel demyelination. The spatio-temporal distribution of action potentials along demyelinated axons and conduction properties including the refractory period and frequency encoding in these two patterns were investigated. The results showed that demyelination could induce the decrease both in the amplitude of action potentials and the ability of frequency coding. Furthermore, the signal conduction velocity in the second-level demyelination was about $21 \%$ slower than that in the first-level demyelination. The refractory period in the second-level demyelination was about $32 \%$ longer than the first-level. Thus, detecting the signal conduction in demyelinated axons by action-potential-encoded optical SHG could greatly improve the assessment of demyelinating disorders to classify the patients. This technique also offers a potential fast and noninvasive optical approach for monitoring membrane potential.
\end{abstract}

Keywords: First-level demyelination; second-level demyelination; demyelinated axons; optical second harmonic generation; action potential.

This is an Open Access article published by World Scientific Publishing Company. It is distributed under the terms of the Creative Commons Attribution 3.0 (CC-BY) License. Further distribution of this work is permitted, provided the original work is properly cited. 


\section{Introduction}

Many optical imaging techniques, including twophoton excitation fluorescence (TPEF), second harmonic generation (SHG) and coherent antiStokes Raman scattering (CARS), enable the specific visualization of the processes related to the neuronal activity. ${ }^{1-3}$ In particular, SHG offers much promise for detecting the transmembrane voltage transients with high temporal and spatial resolution. $^{4-8}$ Dombeck et al. found that SHG technique is better to record the action potential than TPEF with the lipophilic styryl dye FM4- $64 .^{4,5}$ Nuriya et al. carried out SHG imaging of membrane potentials in axons and dendritic spines in cultured hippocampal neurons to study the basic principles of voltage propagation. ${ }^{6,7}$ Voltage abnormalities in demyelinated axon have also been investigated by numerical simulation. The numerical simulation is usually based on the modified Hodgkin-Huxley equations or Frankenhaeuser-Huxley equations. ${ }^{9-12}$ Shneider et al. proposed a model of action potential sensitive SHG to describe the conduction of nerve impulses along a nonmyelinated axon and a myelinated axon. ${ }^{13,14} \mathrm{~A}$ number of studies showed that action-potential-encoded optical SHG is gradually used to examine the axonal damage in demyelinating disorders which are diagnosed mainly by magnetic resonance imaging (MRI) at present. ${ }^{14-18}$ However, MRI is unavailable for distinguishing some demyelinating disorders, such as primary progressive multiple sclerosis (PPMS) and secondary progressive multiple sclerosis (SPMS). ${ }^{17-26}$

In our earlier work, we had simulated the demyelination of a nerve fiber by action-potentialencoded optical SHG. ${ }^{16}$ Here, we extended this approach to a further application to characterize the conduction of pulses along partial demyelinated axons with two types of severity. The models about the first-level demyelination and the second-level demyelination were built via modified HodgkinHuxley equations and cable equations. We focused on the differences in signal conduction between these two models. And these two demyelinated axons could be distinguished by studying the spatiotemporal distribution of action potentials and conduction properties including the refractory period and the frequency coding. Obtaining the properties of signal conduction would be helpful to assess the extent of demyelinating diseases by actionpotential-encoded optical SHG.

\section{Methodology}

In myelinated nerves, axon consists of Ranvier nodes and internodes, as shown in Fig. 1(a). Action potentials generate in the axon initial segment and propagate along the axon from one node of Ranvier to the next, known as saltatory conduction due to the insulation of myelin. In this paper, we built two demyelinated axon models as shown in Fig. 1(b). According to the demyelinating diseases, such as multiple sclerosis, there is much demyelination with the development of the disease. ${ }^{22-26}$ And considering that remyelination also occurred in demyelinated lesions, the internodes would not only thin but also become shorter than normal. ${ }^{23}$ For simplicity, we regarded the first model as the axon of the first-level demyelination as shown in Fig. 1(b) (1) and the second model as the axon of the secondlevel demyelination as shown in Fig. 1(b)(2).

In the section of Ranvier node, there are many ion channels, notably sodium channels, potassium channels and small part of other leak channels. ${ }^{27}$ According to the modified myelinated $\mathrm{HH}$ model, the membrane voltage across the Ranvier node can

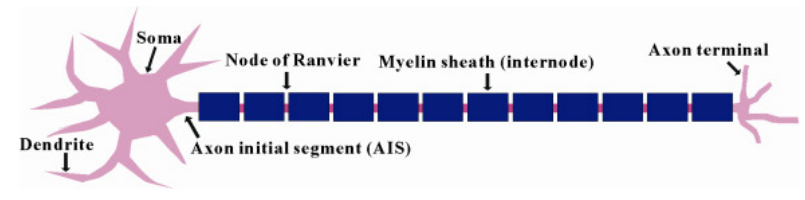

(a)

(1)

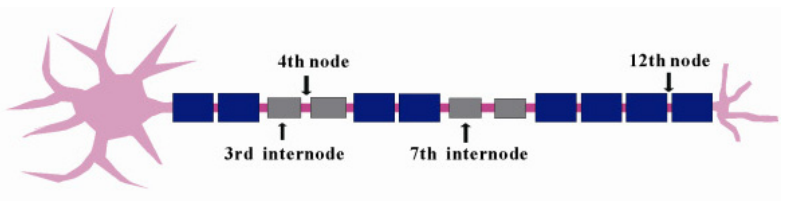

(2)

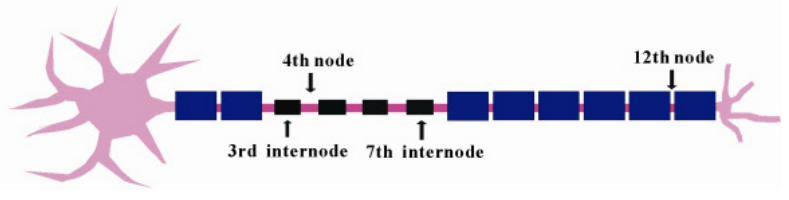

(b)

Fig. 1. Schematic of neurons (Not in scale). (a) A typical neuron; (b) (1) An irregular demyelinated axon with two separated parts of demyelination and the myelin sheath in each part was $0.3 \mu \mathrm{m}$ and $10 \%$ shorter than normal (regarded as the first-level demyelination); (2) A uniformly demyelinated axon with four successive internodal demyelination and the myelin sheath was $0.25 \mu \mathrm{m}$ and $30 \%$ shorter than normal (regarded as the second-level demyelination). 
be described as, ${ }^{9,28}$

$$
\begin{gathered}
C_{m} \frac{\partial V_{r}}{\partial t}=\frac{d}{4 \rho} \frac{\partial^{2} V_{r}}{\partial x^{2}}-\pi l d\left[g_{\mathrm{Na}} m^{3} h\left(V_{r}-V_{\mathrm{Na}}\right)\right. \\
\left.+g_{K} n^{4}\left(V_{r}-V_{\mathrm{K}}\right)+g_{L}\left(V_{r}-V_{L}\right)\right]-I_{\mathrm{stim}}
\end{gathered}
$$

where, $V_{r}$ is the transmembrane potential in the Ranvier node, $m, h$ and $n$ are the relevant gating variables. These gating variables are expressed as functions of $\alpha_{j}$ and $\beta_{j}$, which are the opening rate and closing rate of ion channels, respectively, ${ }^{10,28}$

$$
\begin{gathered}
d j / d t=\phi(T)\left[\alpha_{j}(1-j)-\beta_{j} j\right], \quad(j=m, h, n) \\
\phi(T)=Q_{10}^{(0.1 T-0.63)} \\
\alpha_{m}=Q_{10} \frac{2.5-0.1 V}{\mathrm{e}^{2.5-0.1 V}-1} \quad \beta_{m}=4 Q_{10} \mathrm{e}^{\frac{-V}{18}} \\
\alpha_{n}=Q_{10} \frac{1-0.1 V}{10\left(\mathrm{e}^{1-0.1 V}-1\right)} \quad \beta_{n}=0.125 Q_{10} \mathrm{e}^{\frac{-V}{80}} \\
\alpha_{h}=0.07 Q_{10} \mathrm{e}^{\frac{-V}{20}} \quad \beta_{h}=Q_{10} \frac{1}{\mathrm{e}^{3-0.1 V}+1} .
\end{gathered}
$$

Herein, contrasting to the original model of Hodgkin and Huxley, our model is based on a temperature-dependent coefficient of $Q_{10}=2.2$ for $T=20^{\circ} \mathrm{C} .{ }^{29}$ The opening and closing rates of ion channels depend on membrane voltage and they are also simultaneously influenced by temperature. ${ }^{9,28,29}$

In the section of internode, there is no appreciable ion current passing through the myelin sheath which is an electrical insulator. According to cable model, the voltage difference across the myelin sheath could be expressed as, ${ }^{14,30}$

$$
\frac{k_{1}}{\ln (D / d)} \frac{\partial V_{s}}{\partial t}=\frac{\pi d^{2}}{4 \rho} \frac{\partial^{2} V_{s}}{\partial x^{2}}-\frac{V_{s}}{k_{2} \cdot \ln (D / d)} .
$$

For simplification, the demyelination would be considered to decrease the numerical ratio between the axonal diameter with myelin sheath and the axonal diameter without myelin sheath. Then the term $k_{1} / \ln (D / d)$ representing the myelin capacitance per unit length would increase and the term $k_{2} \cdot \ln (D / d)$ representing the myelin resistance per unit length would decrease. Thus, the dynamics and properties of the membrane potentials would change. Relative parameters in our model are collected in Table 1.

In order to detect the dynamics and properties of the membrane potentials in axons by SHG, a fast optical probe, FM4-64, was assumed to be located inside the neuron membrane. The SHG signal intensity could be modulated linearly by electric field and the relationship between SHG signal intensity and electric field could be described as, ${ }^{13,15}$

$$
I^{\mathrm{SHG}}(E)=I_{0}^{\mathrm{SHG}}[1+\kappa E(1-\theta)] .
$$

Herein, $I^{\mathrm{SHG}}$ is the SHG signal intensity as action potentials passed while $I_{0}^{\text {SHG }}$ represents the SHG signal intensity in resting potential. Set $\kappa=$ $7 \times 10^{-12} \mathrm{~m} / \mathrm{mV}, \theta=0.26$, when the membrane was irradiated by an 800 -nm laser. ${ }^{13,14}$

Table 1. List of parameters of a normal myelinated axon model.

\begin{tabular}{cll}
\hline Parameter & \multicolumn{1}{c}{ Description } & \multicolumn{1}{c}{ Value } \\
\hline$D$ & Axonal diameter with myelin sheath & $15 \mu \mathrm{m}$ [Refs. 14, 15] \\
$d$ & Axonal diameter without myelin sheath & $10 \mu \mathrm{m}$ [Refs. 14, 15] \\
$L$ & Length of the internode & $2 \mathrm{~mm}[$ Refs. 14, 15] \\
$l$ & Length of the Ranvier node & $2.5 \mu \mathrm{m}[$ Refs. 14, 15] \\
$C_{m}$ & Membrane capacitance & $1 \times 10^{-6} \mathrm{~F} / \mathrm{cm}^{2}[$ Refs. 12, 13] \\
$g_{\mathrm{Na}}$ & Conductance of sodium & $120 \mathrm{mS} / \mathrm{cm}^{2}[$ Refs. 9, 13] \\
$g_{K}$ & Conductance of potassium & $36 \mathrm{mS} / \mathrm{cm}^{2}[$ Refs. 9, 13] \\
$g_{L}$ & Conductance of leakage & $0.3 \mathrm{mS} / \mathrm{cm}^{2}[$ Refs. 9, 13] \\
$V_{\mathrm{Na}}$ & Battery voltage of sodium & $115 \mathrm{mV} \mathrm{V}^{9}$ \\
$V_{\mathrm{K}}$ & Battery voltage of potassium & $-12 \mathrm{mV}$ \\
$V_{L}$ & Battery voltage of leakage & $10.6 \mathrm{mV}$ \\
$\rho$ & Specific resistance of the axoplasm & $110 \Omega \cdot \mathrm{cm}^{9}[$ Ref. 14] \\
$m$ & Sodium activation & $0.05^{9}[$ Initial value, Dimensionless] \\
$h$ & Sodium inactivation & $0.6^{9}\left[\mathrm{Initial} \mathrm{value,} \mathrm{Dimensionless]}^{9}\right.$ \\
$n$ & Potassium activation & $0.32^{9}[$ Initial value, Dimensionless] \\
\hline
\end{tabular}




\section{Z.-H. Luo et al.}

The electric field at the node of Ranvier could be expressed as, ${ }^{7,8}$

$$
E\left(x_{r}, t\right)=\frac{V_{r}(t)}{\delta}
$$

where, $\delta$ is the thickness of the bilayer lipid membrane.

Thus, from the Eqs. (6) and (7), the relationship between the differential SHG signal intensity $\left(\Delta I^{\mathrm{SHG}} / I_{0}^{\mathrm{SHG}}\right)$ and the membrane potential $\left(V_{r}(t)\right)$ is linear.

\section{Results and Discussion}

Some key characteristics of a normal action potential are listed in Table 2, including rising phase, falling phase, spike width, amplitude and velocity. These characteristics will help to assess the production and conduction of action potential on demyelinated axons. The duration of the rising phase was about $0.17 \mathrm{~ms}$ which was similar to Carpio's and Smit's works. ${ }^{14,31}$ Likewise, the spike

Table 2. The characteristics of a single normal action potential.

\begin{tabular}{lcc}
\hline Characteristics & Our work & Other researchers' works \\
\hline Rising phase (ms) & 0.17 & $0.12-0.27^{14,31}$ \\
Falling phase (ms) & 0.85 & $0.75^{14}$ \\
& & $0.78^{31}$ \\
Spike width (ms) & 0.56 & $0.62^{32}$ \\
Amplitude (mV) & 105.4 & $90-120^{14,32}$ \\
Velocity (m/s) & 50.6 & $50^{34}$ \\
\hline
\end{tabular}

width also fell in the range of 0.14 to $4 \mathrm{~ms}$ measured by Bean et al., ${ }^{32}$ and the velocity fell in the normal range of 10 to $60 \mathrm{~m} / \mathrm{s}^{33}$

\subsection{Spatio-temporal characteristics of action potentials conduction along axons of the first-level and second-level demyelination}

Figure 2 shows the spatio-temporal distribution of action potentials conducting along the different axons. From Fig. 2(a), we could find that the differential SHG signal intensity decreased in both axons of the first-level and second-level demyelination, while it did not change in normal axon. Furthermore, the signal intensity in node 6 could increase again in the first-level demyelinated axon for the normal myelin sheath at internodes 5 and 6 . While in the second-level demyelinated axon, the signal intensity decreased uniformly in successive internodes demyelination. A comparison of individual waveforms developed at node 4 of these axons is shown in Fig. 2(b). An important finding was that the signal intensity in the second-level demyelination had a relatively slowest rising slope and a significant longest duration compared to that in the first-level demyelination and normal axon. These findings indicated that demyelination could effectively decrease the amplitude, slow the rising slope and prolong the duration of action potential. Furthermore, the rising slope and the duration of action potentials in the second-level demyelination were smaller and longer than that the first-level one.

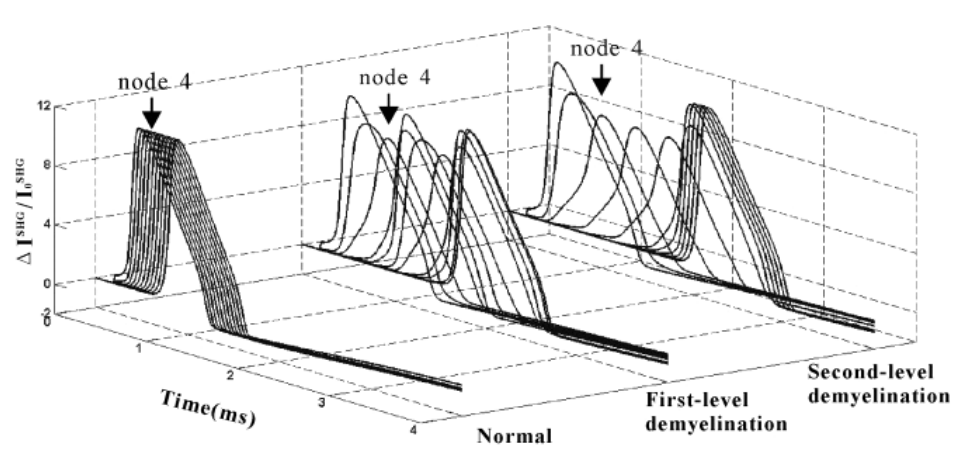

(a)

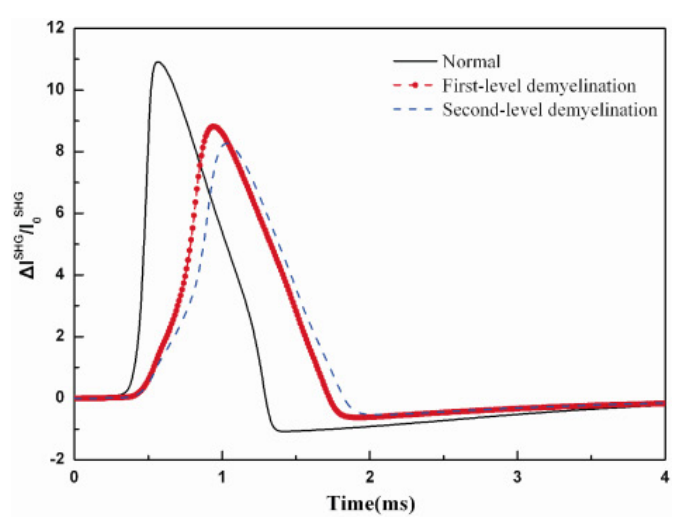

(b)

Fig. 2. Spatio-temporal distribution of the differential SHG signal depending on action potential. (a) Action potentials from node 2 through node 12 in axons of normal, the first-level demyelination and the second-level demyelination; (b) Comparison of individual waveforms developed in the node 4 in axons of normal, the first-level demyelination and the second-level demyelination. 
One reason may be that higher capacitance due to the loss of the myelin sheath needs a greater time constant which could result in an increased conduction delay. ${ }^{28}$ Another reason may be that its lower resistance due to the loss of myelin sheath leads to uniformly dissipated transmembrane currents over the demyelinated internode resulting in a voltage attenuation in Ranvier node. ${ }^{28}$ Similar results have been obtained by Shneider's and Koles' groups. ${ }^{10,14}$

To compare the difference in the velocity of the membrane potential conduction along the first-level and second-level demyelinated axons in more detail, we tracked the peak of voltage from node 2 to node 12, as presented in Fig. 3. The maximal voltages in each node in normal axon were close together with a uniform distribution, and the conduction velocity was about $50.6 \mathrm{~m} / \mathrm{s}$ while in the first-level demyelinated axon, the amplitudes decreased most in node 4 and node 8 with a shape of undulation. It is worth noticing that the time lag from node 2 to node 12 was more than three times longer than the normal one and the conduction velocity was about $15.6 \mathrm{~m} / \mathrm{s}$. In the second-level demyelinated axon, the amplitudes decreased most in the middle nodes in demyelinated region. The time lag was approximately four times longer than normal axon and the conduction velocity was about $12.3 \mathrm{~m} / \mathrm{s}$. As a result, the signal conduction velocity in the second-level demyelination was about $21 \%$ slower than the first-level one. Similar results were

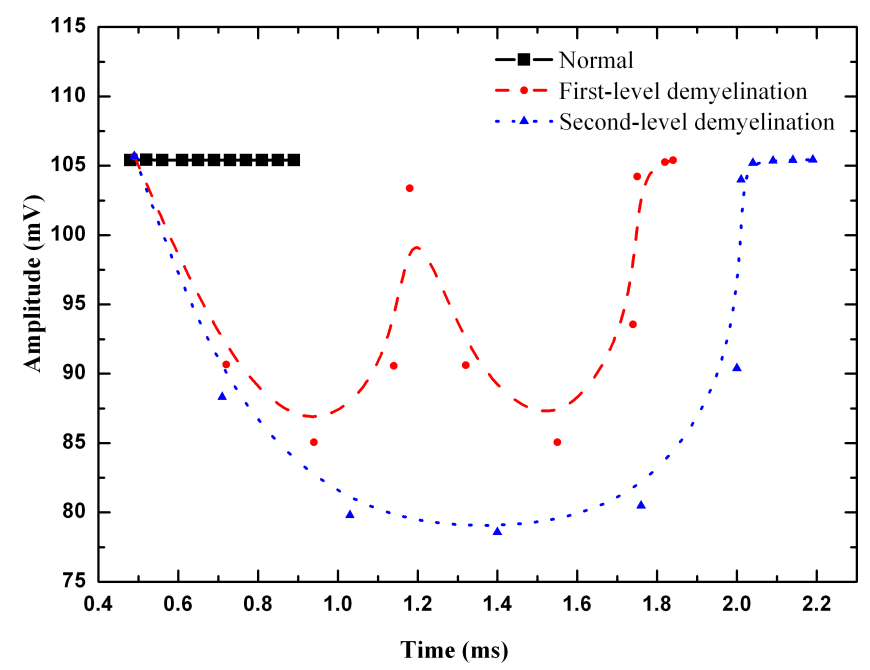

Fig. 3. The distribution of voltage peaks from node 2 to node 12 in axons of normal, the first-level and second-level demyelination. obtained by the group of Stephanova. ${ }^{35}$ In general, it is more capable to acquire a higher potential level when the myelin sheath is thicker. These results also highlighted the importance of myelin sheath to signal conduction. ${ }^{33}$ By observing the spatial and temporal characteristics of action potential and estimating the signal conduction velocity in central nervous system neurons in patients, we might compare the extent of demyelinating disease to distinguish patients with primary progress and secondary progress.

\subsection{Conduction properties in axons of the first-level and second-level demyelination}

\subsubsection{The refractory period}

To better evaluate the mechanisms of demyelination, we study the refractory period besides the actionpotential amplitude and conduction velocity. Figure 4 illustrated the comparison of refractory period between normal axon, the first-level and second-level of demyelinated axons. In the normal axon, when the interval of pulses was less than $1.8 \mathrm{~ms}$, there was almost no action potential at node 12 to respond to the second stimulus. Whereas, when the interval of pulses was $1.8 \mathrm{~ms}$, even larger, the action potential at node 12 could be developed to respond to the second stimulus. By the same token, in the first-level and second-level demyelinated axon, when the interval of pulses was greater than

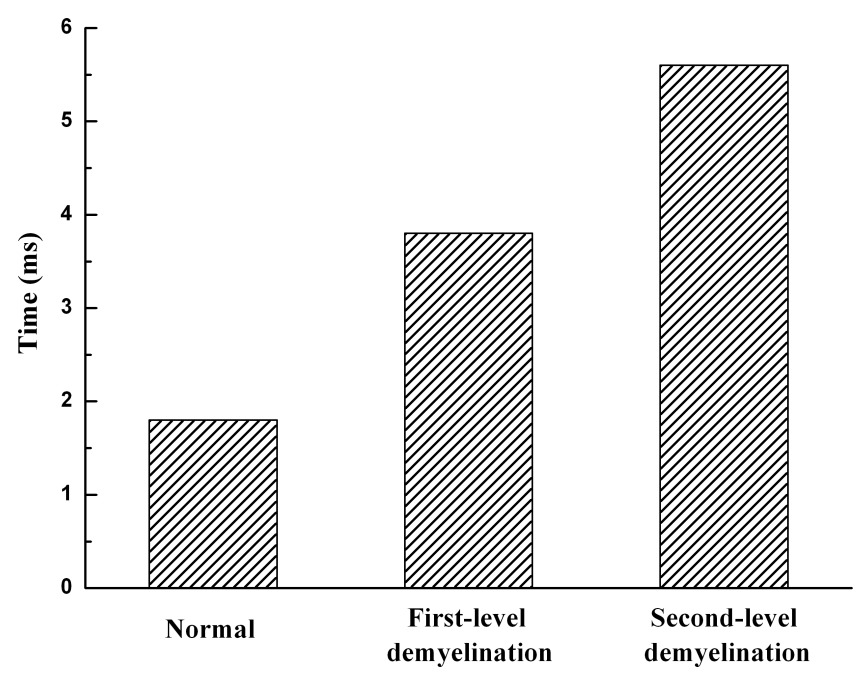

Fig. 4. The refractory periods of axons of normal, the firstlevel and second-level demyelination. 
or equal to 3.8 and $5.6 \mathrm{~ms}$, respectively, the action potential at node 12 could be developed. In other words, the refractory period of the second-level demyelination could be up to $32 \%$ longer than the first-level one. The groups of Felts and Quandt also found that demyelination could induce an extended refractory period. ${ }^{12,36}$ One reason may be that first pulse conducting slowly through the demyelination region avoided the second pulse following behind closely. $^{12}$ Therefore, checking on the refractory period might be an alternative useful method for distinguishing the patients with similar clinic symptoms of demyelinating diseases, such as PPMS and SPMS.

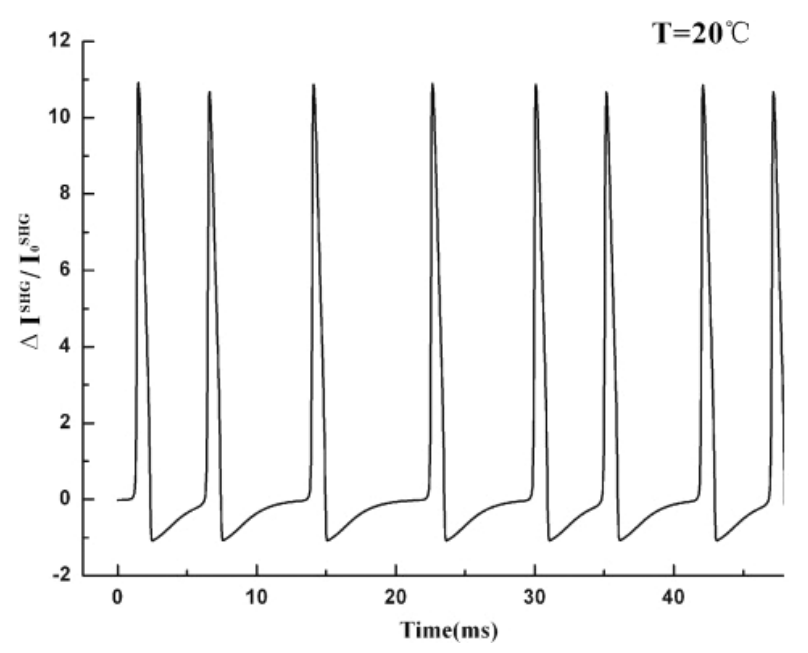

(a)

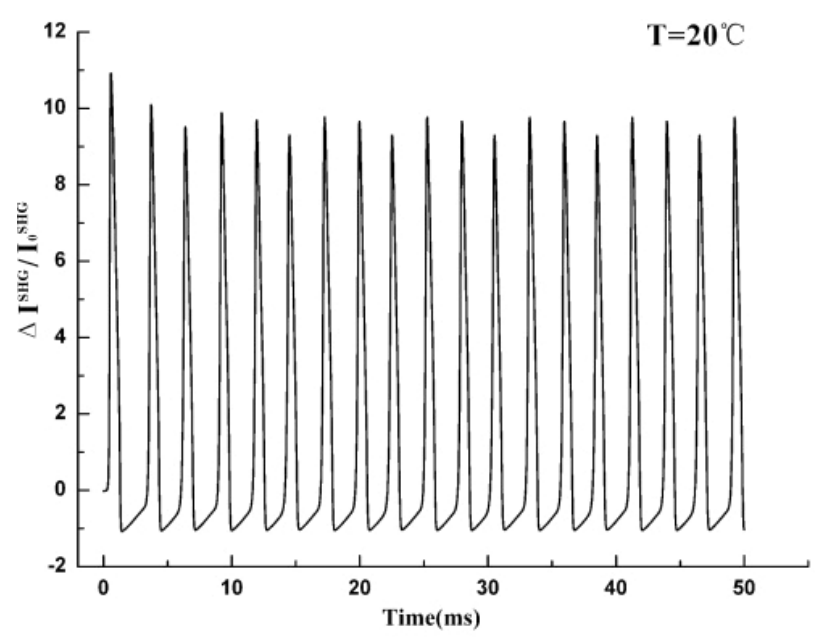

(c)

\subsubsection{Frequency encoding}

One of the fundamental properties of a neuron is to allow stimulus signals to be encoded into the firing frequency of neurons. In order to investigate the ability of frequency encoding in the first-level and second-level of demyelinated axons, we applied a train of suprathreshold stimuli to the axons at the first node. Figure 5 depicted the spike frequency accommodation at node 12 by action-potentialencoded optical SHG in the same stimulus frequency. Over the same period of $50 \mathrm{~ms}$, total amount of visual action potential in the first-level demyelinated axon was 8 which could be almost one-third more than that the second-level one, see

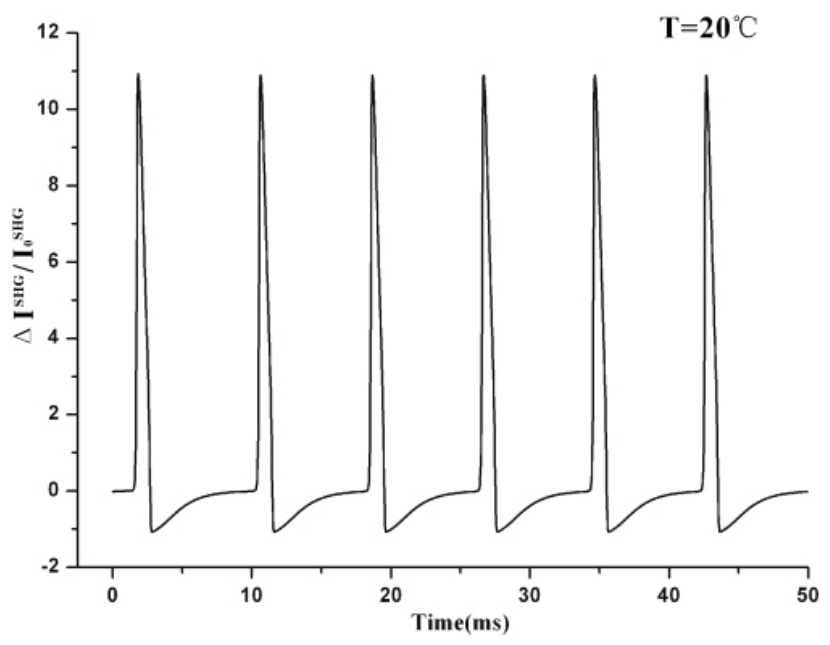

(b)

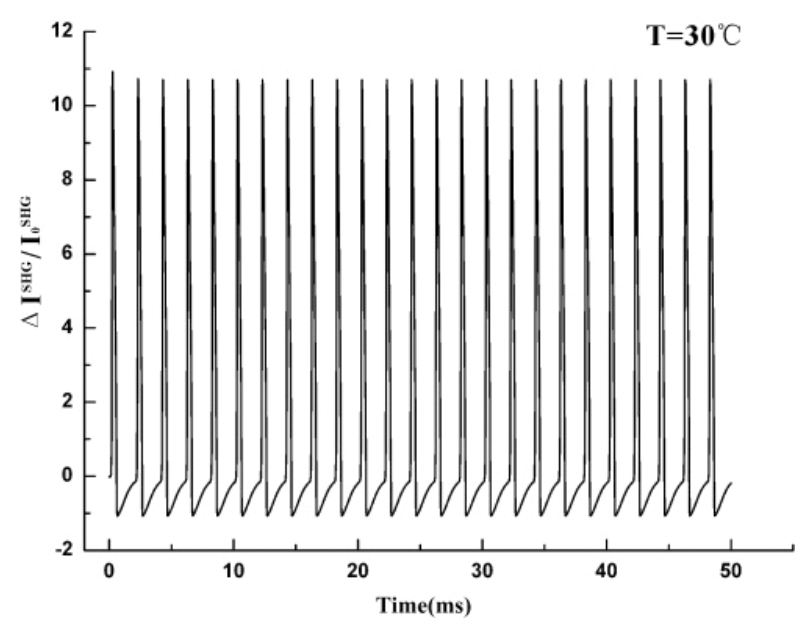

(d)

Fig. 5. Spike frequency accommodation at node 2 by action-potential-encoded SHG in the same stimulus frequency. (a) An axon of the first-level demyelination; (b) A axon of the second-level demyelination; (c) A normal nerve axon; (d) A normal nerve axon with a higher temperature of $30^{\circ} \mathrm{C}$. 
Figs. 5(a) and 5(b), while the total amount of visual action potential in normal axon could be as large as 19, as depicted in Fig. 5(c). In addition, Fig. 5(d) showed the spike frequency accommodation at a higher temperature of $30^{\circ} \mathrm{C}$ in normal axon, 25 spikes were counted. In other words, the ability to conduct repetitive impulses in demyelinated neuron was decreased. Moreover, this ability in the second-level demyelination was even weaker than the first-level demyelination. However, it would be even stronger when the temperature increased in a proper range. An important cause for this critical behavior was the prolonged actionpotential duration and the refractory period in demyelinated region as mentioned above. Then depolarization was counteracted by hyperpolarization that did not satisfy the threshold voltage, therefore, it could filter some following closed pulses. $^{37,38}$ Thus, detecting the frequency encoding by the sensitive optical SHG technology could greatly improve the assessment of demyelinating disorders rapidly.

\section{Conclusions}

Signal conduction properties in the first-level demyelinated axon and the second-level demyelinated axon were investigated by action-potentialencoded optical SHG. We found that demyelination could induce less amplitude, slower rising depolarization and longer duration of action potential. Furthermore, the velocity in axon of the secondlevel demyelination was about $21 \%$ slower than that in first-level one. And in the second-level demyelination, the ability to conduct repetitive impulses was weaker and the refractory period was prolonged to about $32 \%$ than that for the first-level demyelination. In conclusion, primary progress and secondary progress of demyelinating diseases with similar clinic symptoms might be distinguished by investigating the spatio-temporal distribution of action potentials and the conduction properties in demyelinated axons by action-potential-encoded SHG. This technique could be a potential tool for monitoring membrane potential and helps to improve the understanding and assessment of demyelinating disorders.

\section{Acknowledgments}

This work was supported by the National Nature Science Foundation of China under Grant No. 61335011, Program for Changjiang Scholars and Innovative Research Team in University under Grant No. IRT1115 and the Fund from Fujian Normal University under Grant No. 2008100218.

\section{References}

1. H. Segawa, M. Okuno, H. Kano, P. Leproux, V. Couderc, H. Hamaguchi, "Label-free tetra-modal molecular imaging of living cells with CARS, SHG, THG and TSFG (coherent anti-Stokes Raman scattering, second harmonic generation, third harmonic generation and third-order sum frequency generation)," Opt. Express 20(9), 9551-9557 (2012).

2. A. Wunder, J. Klohs, U. Dirnagl, "Non-invasive visualization of CNS inflammation with nuclear and optical imaging," Neuroscience 158(3), 1161-1173 (2009).

3. B. A. Wilt, L. D. Burns, E. T. W. Ho, K. K. Ghosh, E. A. Mukamel, M. J. Schnitzer, "Advances in light microscopy for neuroscience," Annu. Rev. Neurosci. 32(435), 1-79 (2009).

4. D. A. Dombeck, M. Blanchard-Desce, W. W. Webb, "Optical recording of action potentials with secondharmonic generation microscopy," J. Neurosci. 24 (4), 999-1003 (2004).

5. D. A. Dombeck, L. Sacconi, M. Blanchard-Desce, W. W. Webb, "Optical recording of fast neuronal membrane potential transients in acute mammalian brain slices by second-harmonic generation microscopy," J. Neurophysiol. 94(5), 3628-3636 (2005).

6. M. Nuriya, J. Jiang, B. Nemet, K. B. Eisenthal, R. Yuste, "Imaging membrane potential in dendritic spines," Proc. Natl. Acad. Sci. 103(3), 786-790 (2006).

7. M. Nuriya, M. Yasui, "Membrane potential dynamics of axons in cultured hippocampal neurons probed by second-harmonic-generation imaging," $J$. Biomed. Opt. 15(2), 020503 (2010).

8. B. A. Nemet, V. Nikolenko, R. Yuste, "Second harmonic imaging of membrane potential of neurons with retinal," J. Biomed. Opt. 9(5), 873-881 (2004).

9. A. L. Hodgkin, A. F. Huxley, "A quantitative description of membrane current and its application to conduction and excitation in nerve," J. Physiol. 117(4), 500-544 (1952). 
10. Z. J. Koles, M. Rasminsky, "A computer simulation of conduction in demyelinated nerve fibres," $J$. Physiol. 227, 351-364 (1972).

11. Y. G. Yu, Y. S. Shu, D. A. McCormick, "Cortical action potential backpropagation explains spike threshold variability and rapid-onset kinetics," J. Neurosci. 28(29), 7260-7272 (2008).

12. F. N. Quandt, F. A. Davis, "Action potential refractory period in axonal demyelination: A computer simulation," Biol. Cybern. 67(6), 545-552 (1992).

13. M. N. Shneider, A. A. Voronin, A. M. Zheltikov, "Action-potential-encoded second-harmonic generation as an ultrafast local probe for nonintrusive membrane diagnostics," Phys. Rev. E 81(3), 031926 (2010).

14. M. N. Shneider, A. A. Voronin, A. M. Zheltikov, "Modeling the action-potential-sensitive nonlinearoptical response of myelinated nerve fibers and short-term memory," J. Appl. Phys. 110(9), 094702 (2011).

15. H. Q. Yang, X. G. Chen, Y. M. Huang, Z. H. Luo, H. Li, S. S. Xie, "Membrane potential dynamics of nerve fibers fast probed by action-potential-encoded second harmonic generation," Acta Opt. Sin. 32(4), 0417001 (2012).

16. H. Q. Yang, Z. H. Luo, X. G. Chen, Y. M. Huang, S. S. Xie, "Simulating the demyelination of a nerve fiber by action potential encoded second harmonic generation," Proc. SPIE 8553, 85530Z-1-9 (2012).

17. C. Lebrun, C. Bensa, M. Debouverie, S. Wiertlevski, D. Brassat, J. D. Seze, L. Rumbach, J. Pelletier, P. Labauge, B. Brochet, A. Tourbah, P. Clavelou, "Association between clinical conversion to multiple sclerosis in radiologically isolated syndrome and magnetic resonance imaging, Cerebrospinal Fluid, and Visual Evoked Potential," Arch. Neurol. 66(7), 841-846 (2009).

18. C. H. Polman, S. C. Reingold, B. Banwell, M. Clanet, J. A. Cohen, M. Filippi, K. Fujihara, E. Havrdova, M. Hutchinson, L. Kappos, F. D. Lublin, X. Montalban, P. O'Connor, M. Sandberg-Wollheim, A. J. Thompson, E. Waubant, B. Weinshenker, J. S. Wolinsky, "Diagnostic criteria for multiple sclerosis: 2010 revisions to the McDonald criteria," Ann. Neurol. 69(2), 292-302 (2011).

19. L. Mayo, F. J. Quintana, H. L. Weiner, "The innate immune system in demyelinating disease," Immunol. Rev. 248(1), 170-187 (2012).

20. R. Dutta, B. D. Trapp, "Mechanisms of neuronal dysfunction and degeneration in multiple sclerosis," Prog. Neurobiol. 93(1), 1-12 (2011).
21. C. Lucchinetti, W. Bruck, "The pathology of primary progressive multiple sclerosis," Mult. Scler. 10(3), S23-S30 (2004).

22. A. Kutzelnigg, C. F. Lucchinetti, C. Stadelmann, W. Brück, H. Rauschka, M. Bergmann, M. Schmidbauer, J. E. Parisi, H. Lassmann, "Cortical demyelination and diffuse white matter injury in multiple sclerosis," Brain 128(11), 2705-2712 (2005).

23. S. Bramow, J. M. Frischer, H. Lassmann, N. KochHenriksen, C. F. Lucchinetti, P. S. Sørensen, H. Laursen, "Demyelination versus remyelination in progressive multiple sclerosis," Brain 133(10), 2983-2998 (2010)

24. J. M. Margolis, R. Fowler, B. H. Johnson, C. A. Kassed, K. Kahler, "Disease-modifying drug initiation patterns in commercially insured multiple sclerosis patients: A retrospective cohort study," BioMed Central Neurology. 11(122), 1-10 (2011).

25. B. D. Trapp, K. Nave, "Multiple Sclerosis: An immune or neurodegenerative disorder?" Annu. Rev. Neurosci. 31, 247-269 (2008).

26. J. J. G. Geurts, F. Barkhof, "Grey matter pathology in multiple sclerosis," Lancet Neurol. 7(9), 841-851 (2008).

27. D. Debanne, E. Campanac, A. Bialowas, E. Carlier, G. Alcaraz, "Axon physiology," Physiol. Rev. 91(2), 555-602 (2011).

28. A. Carpio, I. Peral, "Propagation failure along myelinated nerves," J. Nonlinear Sci. 21(4), 499520 (2011).

29. J. F. Fohlmeister, E. D. Cohen, E. A. Newman, "Mechanisms and distribution of ion channels in retinal ganglion cells: Using temperature as an independent variable," J. Neurophysiol. 103(3), 1357-1374 (2010)

30. C. C. McIntyre, A. G. Richardson, W. M. Grill, "Modeling the excitability of mammalian nerve fibers: Influence of afterpotentials on the recovery cycle," J. Neurophysiol. 87(2), 995-1006 (2002).

31. J. E. Smit, T. Hanekom, J. J. Hanekom, "Modelled temperature-dependent excitability behaviour of a single ranvier node for a human peripheral sensory nerve fibre," Biol. Cybern. 100(1), 49-58 (2009).

32. B. P. Bean, "The action potential in mammalian central neurons," Nat. Rev. Neurosci. 8, 451-465 (2007).

33. S. G. Waxman, "Determinants of conduction velocity in myelinated nerve fibers," Muscle Nerve $\mathbf{3}$ (2), 141-150 (1980).

34. C. H. Fry, R. I. Jabr, "The action potential and nervous conduction," Surgery (Oxford) 28(2), 49-54 (2010). 
35. D. I. Stephanova, M. Daskalova, A. S. Alexandrov, "Differences in potentials and excitability properties in simulated cases of demyelinating neuropathies Part I," Clin. Neurophysiol. 116(5), 1153-1158 (2005).

36. P. A. Felts, T. A. Baker, K. J. Smith, "Conduction in segmentally demyelinated mammalian central axons," J. Neurosci. 17(19), 7267-7277 (1997).
37. H. Bostock, P. Grafe, "Activity-dependent excitability changes in normal and demyelinated rat spinal root axons," J. Physiol. 365, 239-257 (1985).

38. S. Kuwabara, Y. Nakajima, T. Hattori, S. Toma, K. Mizobuchi, K. Ogawara, "Activity-dependent excitability changes in chronic inflammatory demyelinating polyneuropathy: A microneurographic study," Muscle Nerve 22(7), 899-904 (1999). 